\title{
Measurement of the Rate of Water Translocation through Carbon Nanotubes
}

\author{
Xingcai Qin, ${ }^{\dagger}$ Quanzi Yuan, ${ }^{\ddagger}$ Yapu Zhao, ${ }^{\ddagger}$ Shubao Xie, ${ }^{\dagger}$ and Zhongfan Liu ${ }^{*,+}$ \\ ${ }^{\dagger}$ Center for Nanochemistry, Beijing National Laboratory for Molecular Sciences, State Key Laboratory for Structural Chemistry of \\ Unstable and Stable Species, College of Chemistry and Molecular Engineering, Peking University, Beijing 100871, China \\ ${ }^{\ddagger}$ State Key Laboratory of Nonlinear Mechanics, Institute of Mechanics, Chinese Academy of Sciences, Beijing 100190, China
}

Supporting Information

ABSTRACT: We present an approach for measuring the water flow rate through individual ultralong carbon nanotubes (CNTs) using field effect transistors array defined on individual tubes. Our work exhibits a rate enhancement of $882-51$ and a slip length of 53-8 $\mathrm{nm}$ for CNTs with diameters of $0.81-1.59 \mathrm{~nm}$. We also found that the enhancement factor does not increase monotonically with shrinking tube diameter and there exists a discontinuous region around $0.98-1.10 \mathrm{~nm}$. We

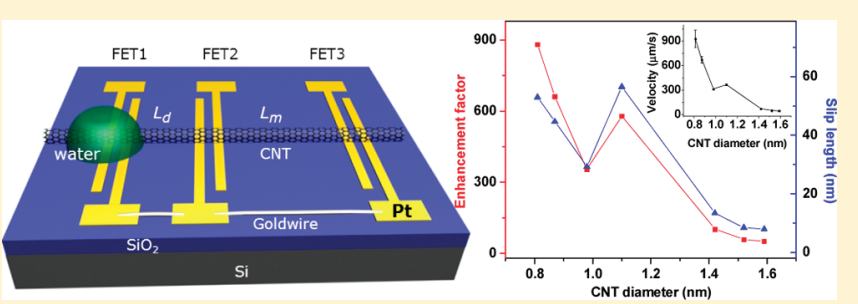
believe that these single-tube level results would help understand the intrinsic nanofluidics of water in CNTs.

KEYWORDS: Nanofluidics, water flow velocity, enhancement factor, slip length, CNT-FET

Ghanced water flow through atomic smooth and hydrophoEbic carbon nanotubes (CNTs) have been demonstrated by both theoretical calculations and experiments. ${ }^{1-5}$ There is, however, a great controversy between theory and experiments and even between experiments. The very limited experiments using CNTs membrane demonstrated enormous water flow velocity up to 5 orders of magnitude faster than predicted from conventional fluid-flow theory with three orders of deviation from different sources. ${ }^{1,2}$ In contrast, molecular dynamics (MD) calculation only gives a rate enhancement of 47-6500 for CNTs with diameters of $4.99-0.81 \mathrm{~nm} .{ }^{3,5}$ One more general debate is whether there exists a clear transition from continuum to subcontinuum transport as the tube diameter shrinks to subnanometer regime. ${ }^{5}$ The bottleneck for experimental attempts arises from fabrication of CNTs membrane with well-defined structures and the rational estimation of the available flow area. ${ }^{1}$ Here we show a single-tube level approach for elucidating such fundamental nanofluidic issues. The unique field effect transistors (FETs) array-based experimental design enables a direct measurement of water flow velocity inside individual CNTs. Our work demonstrates a rate enhancement of 51 to 882 for CNTs with diameters of 1.59 to $0.81 \mathrm{~nm}$, which supports the MD calculation. $^{3,5}$ Additionally, we achieved the first experimental evidence for the transition from continuum to subcontinuum flow by varying the diameters of CNTs.

The key of our approach is to trace the water flow "front" inside an individual millimeter long CNT electrically with a configuration of three FETs in series (Figure 1a,b). The FET1 is used to "in-situ" open the tube end under water droplet by electrical breakdown, ${ }^{6,7}$ and the synchronous FET2 and FET3 to detect the water front flowing in based on its influence on the current flow (Figure 2) ${ }^{8,9}$ It should be emphasized that opening the tube end under water is a determinant factor for the success of this experimental design. A bias voltage of $0.01 \mathrm{~V}$ was applied on FET2 and FET3 (no gate voltage) all the time to detect current change. Simply by measuring the time delay of current signal jumps between FET2 and FET3 with a given interspacing, we can then estimate the average water flow velocity inside the nanotube. The CNT-FETs structure was constructed through directly growing ultralong $\mathrm{CNT}$ on $\mathrm{SiO}_{2} / \mathrm{Si}$ substrate with predesigned Pt-pattern (Figure 1b,c). Carbon nanotubes were synthesized by gas flow-directed chemical vapor deposition (CVD) method. ${ }^{10-12}$ The catalysts pattern was made on growth substrate using PDMS stamp from the ethanol solution of $0.01 \mathrm{~mol} / \mathrm{L} \mathrm{FeCl}_{3}$. The typical growth conditions are $930-950{ }^{\circ} \mathrm{C}, 3 \mathrm{sccm} \mathrm{CH} \mathrm{CH}_{4}$ and 5 sccm $\mathrm{H}_{2}$. Pt was sputtered and patterned as electrodes on $\mathrm{SiO}_{2} / \mathrm{Si}$ substrate by standard technique of photolithography and magnetron sputtering. The as-grown CNTs were characterized by scanning electron microscopy (SEM) followed by gold-wire wedge bonding, water filling and velocity measurement. A drop of pure water $(18.2 \mathrm{M} \Omega \cdot \mathrm{cm})$ was injected onto FET1 by a $1 \mu \mathrm{L}$ of microsyringe which was fixed on a three dimensionally movable microstage for precisely controlling the drop size and position without disturbing the electrical measurement. Atomic force microscopy (AFM) and multiwavelength Raman spectroscopy (632.8, 514.5, $488 \mathrm{~nm}$ ) were employed to distinguish individual CNTs from bundles and to determine the exact tube structures ${ }^{13,14}$ together with measurements of electrical resistance and saturation current of $\mathrm{CNTs}^{7,13,14}$ (Supporting Information, Figure S1).

For the CNT-FETs array devices with individual CNTs, we observed two different types of current changes on FET2 and FET3 in response to water injection at the open tube end on

Received: March 14, 2011

Revised: March 30, 2011

Published: April 04, 2011 
FET1 (Figure 2). For a smaller diameter CNT, the current shows a decrease (Figure 2a) while for a larger diameter CNT, it shows an increase (Figure $2 \mathrm{~b}$ ) after water injection. $\mathrm{Na}$ et al. have investigated the effect of water on CNT-FETs. ${ }^{8}$ They found that water molecules behave as electron donors to CNTs. For the p-type CNT-FETs, the current decreases under a moderate humidity and starts to increase as the humidity increases over $65 \% .{ }^{8}$ We believe that similar doping effect of water may exist in our system, ${ }^{9}$ which creates the theoretical basis of our approach. For small-diameter tubes, the number of injected water molecules is less than carbon atoms flowing over and hence the water doping can only compensate part of holes in CNT-FET, leading to the decrease of electric current. On the other hand, for largediameter tubes the holes are completely compensated and excess doping creates electron conduction channels. ${ }^{8}$ Obviously, there is a time delay on the sharp current jumps between FET2 and FET3. This can be easily understood by considering the time
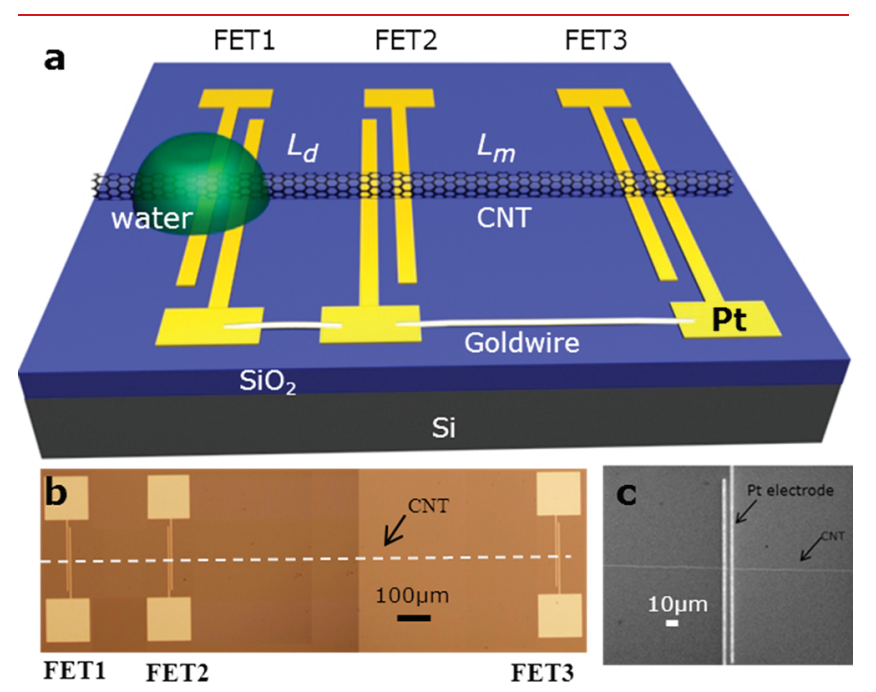

Figure 1. Experimental setup of current-based flow velocity measurement in a CNT. (a) Schematic illustration of the three FETs array device made on one individual CNT with a common ground and a channel length of $6 \mu \mathrm{m}$. The FET1 was used to open the tube end for water filling by electrical breakdown, and the other two FETs to detect the water flow front. The $L_{\mathrm{d}}$ between FET1 and FET2 was for damping down the fast water flow and the flow velocity was obtained as an average in the $L_{\mathrm{m}}$ between FET2 and FET3. (b) Optical microscope image of the typical three CNT-FETs array device, where the dash line indicates the location of CNT. (c) SEM image of FET2 shown in (b). expenses of water flow front arriving to FET2 and to FET3. Therefore, we can estimate the average velocity of water flow between FET2 and FET3 by

$$
v=\frac{L_{\mathrm{m}}}{\left(t_{2}-t_{1}\right)}
$$

where "measure length" $L_{\mathrm{m}}$ is the interspacing between FET2 and FET3, and $t_{1}$ and $t_{2}$ are the arrival times of water flow front to FET2 and FET3, respectively.

To exclude the influence of water flow and/or diffusion along the outer wall of a CNT, two control experiments were performed. First, a hydrophobic photoresist film was spin-coated onto the CNT. As shown in Figure 3a, similar current jumps can be observed on FET2 and FET3 in response to water injection. Second, without opening up the tube end at the site of FET 1 by electrical breakdown, no any current jumps can be observed on the uncovered FET2 and FET3 in response to water injection (Figure $3 \mathrm{~b}$ ). These observations suggest that the current jumps can only be induced by water flow inside the carbon nanotube. To test the reliability of our approach, we cut a millimeter long $\mathrm{CNT}$ into two segments and measured the water flow velocity independently. As can be seen from Table 1, all three different CNTs give reproducible velocity values at two identical segments with a deviation of $<15 \%$. The experimental errors are mainly determined by the "dead" FET conduction channel length (typically a few micrometers) and the time resolution of electrical measurement. To overcome the limitation of system response (typically submilliseconds), the fast water flow can be damped easily by increasing the "damping length" $L_{\mathrm{d}}$ between FET1 and FET2. After systematic optimization of the experimental conditions, we measured the water flow velocity over 50 CNT-FETs array devices, $82 \%$ of which exhibited clear current jumps on FET 2 and FET3 in response to water injection after opening the tube end at FET1. In fact, only those CNTs having small bandgaps were found to be suitable for our nanofluidic measurements, while for large bandgap CNTs no discernible current flow was obtained because of the cutoff effect of FET device. The water flow velocity values experimentally observed fall into a range of $46-928 \mu \mathrm{m} / \mathrm{s}$ for CNTs of $1.59-0.81 \mathrm{~nm}$ in diameter (Figure 5 inset and Supporting Information Table S1).

MD simulations with LAMMPS were used to examine the process of water molecules entering into $(n, n)(n=6-12)$ type of single-walled carbon nanotubes (SWNTs). The MD calculation domain consisted of water molecules and a single-walled carbon nanotube (SWNT), whose diameter ranges from 8.14 to
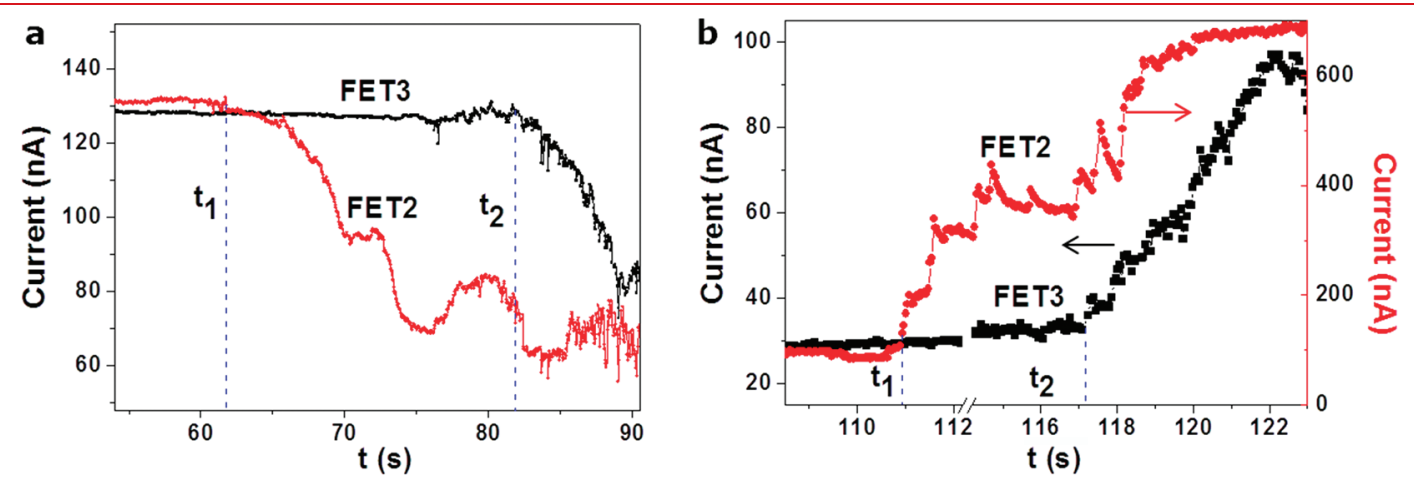

Figure 2. Two types of current changes on FET2 (red) and FET3 (black) in response to water injection at the open tube end on FET1. (a) Current decrease. (b) Current increase. 

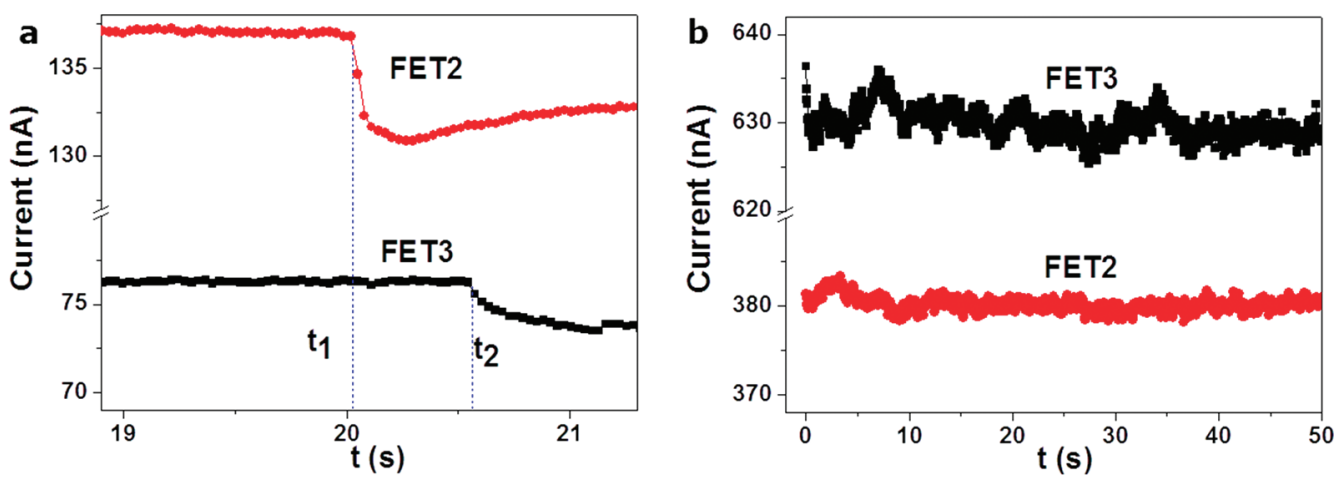

Figure 3. Control experiments for confirming our current-based flow velocity measurement. (a) Current responses on FET2 and FET3 after excluding water flow/diffusion along CNT outer wall by covering CNT with hydrophobic photoresist; (b) current responses on FET2 and FET3 with the uncovered CNT end being not opened at FET1.

Table 1. Reliability Test with Three Different CNTs in Which Each CNT Was Cut into Two Identical Segments for Velocity Measurement

\begin{tabular}{lcccc} 
sample & damping length $(\mu \mathrm{m})$ & measure length $(\mu \mathrm{m})$ & velocity of part $1(\mu \mathrm{m} / \mathrm{s})$ & velocity of part $2(\mu \mathrm{m} / \mathrm{s})$ \\
CNT 1 & 140 & 280 & $20.3 \pm 1.6$ & $18.1 \pm 1.4$ \\
CNT 2 & 140 & 280 & $40.8 \pm 2.9$ & $34.2 \pm 2.6$ \\
CNT 3 & 140 & 280 & $21.2 \pm 1.6$ & $23.0 \pm 0.8$ \\
\hline
\end{tabular}

$16.27 \AA$ and whose length is $28.4 \AA$. The simulation box was $5.2 \times 5.2 \times 5.2 \mathrm{~nm}^{3}$. The Nosé-Hoover thermostat with a timestep of $1 \mathrm{fs}$ was employed to regulate the temperature at $300 \mathrm{~K}$. The systems were replicated periodically in all the three dimensions. The extended single point charge (SPC/E) water model was used to simulate the behaviors of water molecules. The reservoirs were filled with 4000 water molecules. The Lennard-Jones $(\mathrm{LJ})$ parameters for water molecules were $\sigma_{\mathrm{O}-\mathrm{O}}=0.3166 \mathrm{~nm}, \varepsilon_{\mathrm{O}-\mathrm{O}}=0.6500 \mathrm{~kJ} / \mathrm{mol}$, $\sigma_{\mathrm{H}-\mathrm{H}}=0.0000 \mathrm{~nm}$, and $\varepsilon_{\mathrm{H}-\mathrm{H}}=0.0000 \mathrm{~kJ} / \mathrm{mol}$ from the SPC $/ \mathrm{E}$ model. The carbon atoms of tube were modeled as uncharged Lennard-Jones (LJ) particles. The $\mathrm{C}-\mathrm{C}$ bond length was $1.42 \AA$. The LJ parameters for carbon atoms were $\sigma_{\mathrm{C}-\mathrm{C}}=0.3617 \mathrm{~nm}$, $\varepsilon_{\mathrm{C}-\mathrm{C}}=0.1480 \mathrm{~kJ} / \mathrm{mol}$. The values of $\sigma$ and $\varepsilon$ between them were calculated according to the Lorentz-Berthelot rule: $\sigma_{x-y}=\left(\sigma_{x-x}+\right.$ $\left.\sigma_{y-y}\right) / 2$ and $\varepsilon_{x-y}=\left(\varepsilon_{x-x} \varepsilon_{y-y}\right) / 2$. The equations of motion were integrated by using a leapfrog algorithm.

Figure 4 shows the tube diameter dependence of water structure and radial density profile inside a $(n, n)$ tube (Figure S2, Supporting Information). Obviously, together with the increase of tube diameter, water molecules behavior like a single-file chain for $(6,6)$ tube, stacked hexagonal rings for $(9,9)$ tube, and disordered bulklike water for $(12,12)$ tube, respectively, which are consistent with previous reports. ${ }^{5,15}$ The corresponding radial density profiles of water in these SWNTs further depict the remarkable size effect at nanoscale regime. It is no doubt that such kind of size effect would strongly affect the nanofluidics of water in CNTs. As described by Hummer et al., ${ }^{16}$ the driving force of water flow into CNTs arises from the lower chemical potential of tube-trapped water molecules relative to bulk water, and the chemical potential difference can be estimated by

$$
\Delta \mu=R T \ln \frac{\rho_{0}}{\rho}
$$

where $\rho$ and $\rho_{0}$ are the respective water densities in $\mathrm{CNT}$ and in the bulk while the former can be obtained by integrating the corresponding radial density profile shown in Figure $4 \mathrm{~b}-\mathrm{d}$. $R$ and $T$ have their conventional meanings. Figure $4 \mathrm{e}$ reveals the correlation of relative water density $\left(\rho / \rho_{0}\right)$ and the chemical potential difference $(\Delta \mu)$ with tube diameters obtained from MD simulation. Neglecting the minor contribution of tube chirality, ${ }^{17}$ both packing density and chemical potential difference exhibit monotonic decrease together with the increase of tube diameter.

Hagen-Poiseuille equation is usually used to describe the steady-state fluid flow in a tube, a modified version of which, the Washburn equation had proved that Hagen-Poiseuille equation can also be applied to a system where the pressure gradient is changing as a function of time. ${ }^{4,18}$ Velocity from Hagen-Poiseuille $(\mathrm{H}-\mathrm{P})$ equation is

$$
v=\frac{r^{2}}{8 \xi} \frac{\Delta P}{L}
$$

where $r$ is the radius of a nanopore, $\xi$ is the viscosity of water, and $\Delta P / L$ is the pressure gradient along nanotube. For our specific experimental configuration, the average velocity of water flow from FET2 to FET3 can be easily derived from Hagen-Poiseuille equation

$$
v_{\mathrm{av}}=\frac{r^{2} \Delta P}{\left(\frac{L_{\mathrm{m}}}{2}+L_{\mathrm{d}}\right) 8 \xi}
$$

where $\Delta P=-\Delta \mu / V$ is the driving pressure, $V$ is the molar volume of water, $\xi$ is the viscosity of water $\left(10^{-3} \mathrm{~Pa} \cdot \mathrm{s}\right)$, and $r$ is the radius of a nanopore. Equation 4 indicates that the average velocity is equivalent to a velocity at the length of $\left(L \mathrm{~m} / 2+L_{\mathrm{d}}\right)$. For a typical damping length of $280 \mu \mathrm{m}$ and measure length of $1050 \mu \mathrm{m}$, the equivalent length is $805 \mu \mathrm{m}$ (Supporting Information Figure S3). With the calculated velocity data, we can then estimate the enhancement factor, defined as the ratio of observed velocity to that calculated from no-slip continuum Hagen-Poiseuille equation, and slip length, when taking slip-flow into consideration (Supporting Information S3). Slip length was obtained from the following 

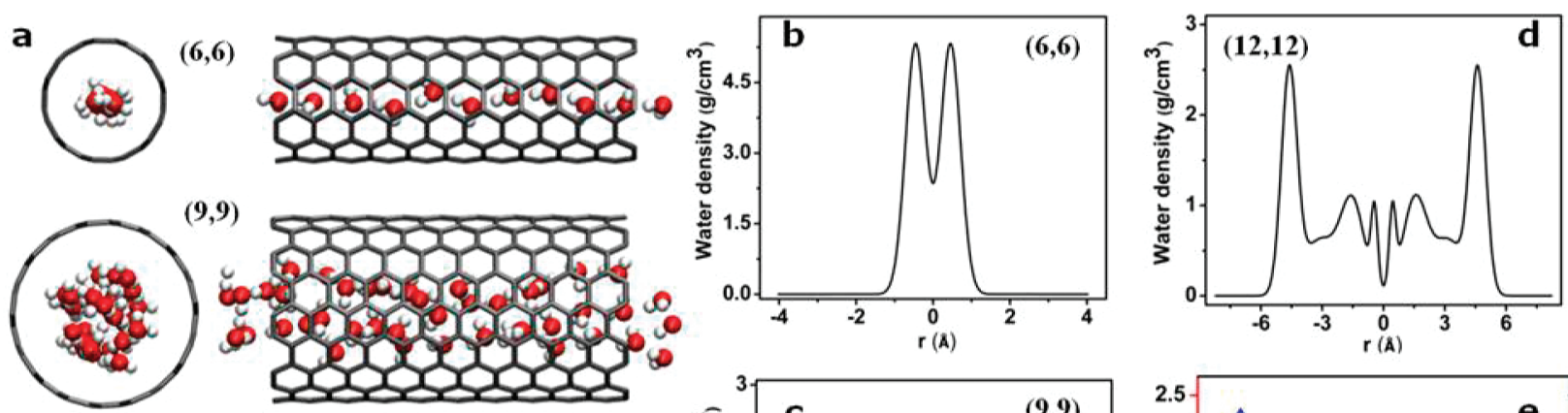

$(12,12)$
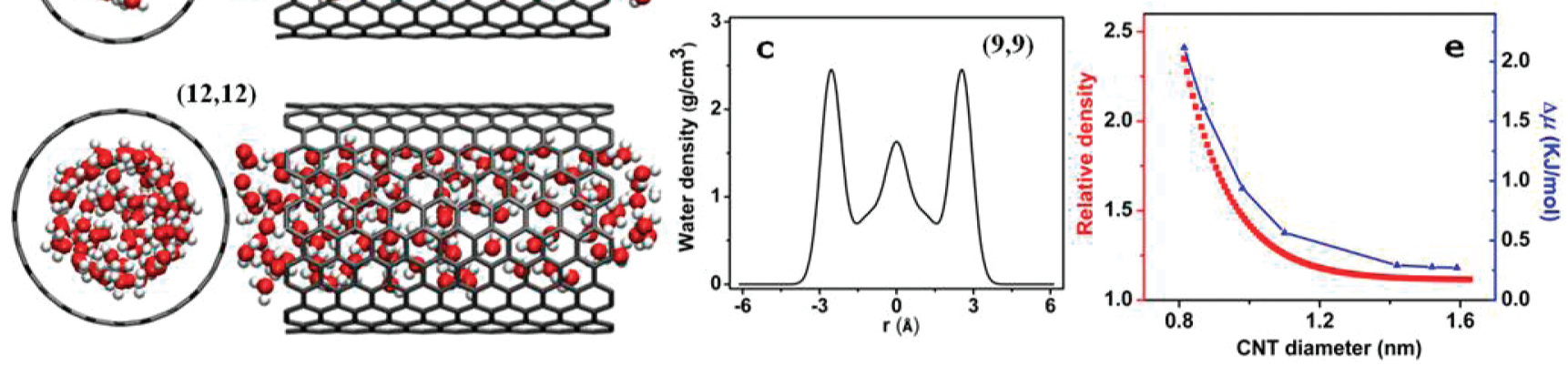

Figure 4. Molecular dynamics simulations on CNT-trapped water molecules. (a) Water structure inside $(6,6)$, $(9,9)$, and $(12,12)$ SWNTs. $(b-d)$ Radial density profiles of water in $(6,6),(9,9)$, and $(12,12)$ SWNT, respectively. The radial distance was toward the wall from the center of the tube at $r=$ $0 \mathrm{~nm}$. (e) CNT-diameter dependences of relative density $\left(\rho / \rho_{0}\right)($ red) and chemical potential difference $(\Delta \mu)$ (blue) of tube-trapped water molecules.

Table 2. Enhancement Factors and Slip Lengths Obtained with Our Single-Tube Approach in Comparison with Previous Theoretical and Experimental Data

diameter of CNT (nm)

our work

J. K. Holt ${ }^{c}$

B. J. Hinds ${ }^{c}$

A. J. H. McGaughey ${ }^{b}$
$0.81-1.59$

$1.3-2.0^{a}$

$7^{a}$

$0.81-1.66$

$1.66-4.99$ enhancement factor

${ }^{c} 51-882$

${ }^{b} 21-192$

560-8400

43860-77018

433-6500

$47-433$ slip length (nm)

${ }^{c} 8-53$

$b_{4-25}$

140-1400

$39000-68000$

$30-105$

${ }^{a}$ Inner pore size of CNT. ${ }^{b}$ Full diameter, modified viscosity was used to calculate enhancement factor and slip length. ${ }^{c}$ Inner pore diameter, invariable viscosity $(1.0 \mathrm{mPa} \cdot \mathrm{s})$ was used for calculation.

equation

$$
v_{\text {slip }}=\frac{r^{2}+4 r L_{s}}{8 \xi} \frac{\Delta P}{L}
$$

Generally, both enhancement factor and slip length are intrinsic to the CNT-water system, which is not sensitive to the experimental conditions such as tube length, pressure and etc.

Table 2 summarizes our measurement results in comparison with previous experimental and theoretical data. ${ }^{1-3,5}$ Instead of flow velocity, we employed enhancement factor and slip length to characterize the intrinsic nanofluidic performance of water-CNT system. To rule out any interference from bundles and multiwalled carbon nanotubes, only those CNTs having well-determined structures were adopted for calculating the enhancement factor and slip length. To our surprise, the enhancement factors fall into a range of 51-882 for CNTs having diameters of $1.59-0.81 \mathrm{~nm}$, which are over 3 orders of magnitude smaller than Hinds's results and about 1 order of magnitude smaller than Holt's results even though our nanotubes have smaller pore sizes. ${ }^{1,2}$ The slip lengths we obtained are from 8 to $53 \mathrm{~nm}$, over 4 orders of magnitude smaller than Hinds's work and 2 orders of magnitude smaller than Holts' work. ${ }^{1,2}$ On the other hand, our single-tube-based approach is well close to
McGaughey's MD simulation. ${ }^{3,5}$ For CNTs having diameters of 4.99- $-0.81 \mathrm{~nm}$, the MD simulation derives a rate enhancement of $47-6500 .{ }^{3,5}$ Our experimental design precluded any uncertainty and averaging effect on determining the actual flow area of CNTs because of the use of single tubes. Complications in our system would be resulted from tube end effects, ${ }^{19}$ structural perfectness, and chirality of CNTs, and possible electrowetting effect. ${ }^{20,21}$ Because of the compensation between different factors and their minor effects on this experiment, ${ }^{19-21}$ it is believed not to change the order of magnitude for the obtained results. Hence the obtained enhancement and slip length values should reflect the intrinsic nanofluidics of water in CNTs. As a conclusion, although the CNTs can provide a fast transport of water, it was seriously overestimated, most probably arising from the difficulties in quantitative nanofluidic measurements.

With this single-tube level approach, we directly achieved the first experimental correlation between nanofluidic behavior and tube diameter at the nanoscale regime (Figure 5). For comparison with previous work, we employed both pore diameter and tube diameter, and both constant viscosity and effective viscosity for calculations, which all gave the similar tendency (Figure S3 Supporting Information). Contrary to the prediction from continuum no-slip Hagen-Poiseuille law, ${ }^{1}$ the flow velocity of water confined in a CNT decreases with increasing the tube diameter 


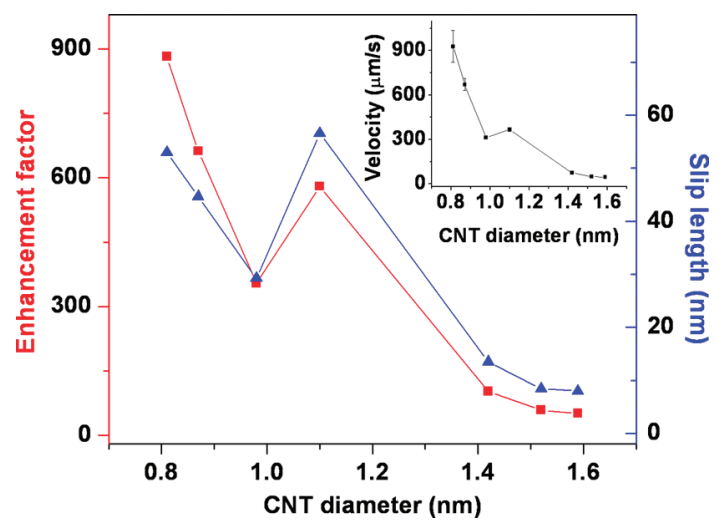

Figure 5. Size effect of enhancement factor (red) and slip length (blue) in CNT-water system in which a pore diameter of CNT and a water viscosity of $1 \mathrm{mPa} \cdot \mathrm{s}$ were used for calculations. The inset plot shows the dependence of experimentally observed water flow velocity (error bar was also showed) on CNT diameter.

(inset of Figure 5). Of particular importance is that the flow enhancement factor does not show a monotonic increase with decreasing tube diameters and there clearly exists a discontinuous region located around $0.98-1.10 \mathrm{~nm}$. This strongly suggests a transition from continuum to subcontinuum flow when the tube diameter shrinks down, well consistent with the theoretical prediction of McGaughey et al. ${ }^{5}$

Although the velocities of water flow obtained from individual SWNTs are remarkably smaller than previous results on CNTs membranes, the enhancement over conventional flow is still very prominent at the nanoscale regime. Especially for $0.81 \mathrm{~nm}$ tubes, over 3 orders of magnitude enhancement has been observed. This certainly reflects the intrinsic nanofluidic property of the CNT-water system, attributable to the atomic level smoothness of tube wall, weak water-tube wall coupling, and the single-file molecular transport phenomenon of water confined in the nanospace of CNTs. ${ }^{1,3,16}$ The discontinuity of enhancement observed in $0.98-1.10 \mathrm{~nm}$ diameter regime, or in other words, the transition of water from continuum to subcontinuum flow, is believed to originate from the structural variation of hydrogen bonding network of water because of spatial confinement effect. Obviously, our approach using single-tubebased electrical measurement would help to elucidate the controversial nanofluidics of water in CNTs though it remains a great challenge to identify the effect of tube chirality and to monitor the extremely fast water flow at the tube inlet because of the limited time resolution of electrical measurements. The present findings would also pave the way for understanding the ion transport through biological channels.

In summary, our single tube level electrical approach based on a configuration of three FETs in series offers an effective way to measure the water flow velocity in individual ultralong carbon nanotubes. The water flow enhancement and slip length in CNTs obtained from our work are orders of magnitude smaller than previously reported using CNTs membrane, but agree well with theoretical prediction. We believe that the previous overestimation comes from the difficult quantitative measurement of effective flow area in CNTs membrane. Therefore our single-tube approach would help to understand the intrinsic nanofluidics of water in CNTs.

\section{ASSOCIATED CONTENT}

S Supporting Information. Growth of ultralong individual CNTs, fabrication of CNT-FETs, molecular dynamics simulations, calculation of enhancement factor and slip length, and pore diameter and viscosity. This material is available free of charge via the Internet at http://pubs.acs.org.

\section{AUTHOR INFORMATION}

\section{Corresponding Author}

*E-mail: zfliu@pku.edu.cn.

\section{ACKNOWLEDGMENT}

We thank the financial support from the National Science Foundation of China (nos. 20973013, 51072004, and 50821061, 60936001, 11072244, and 11021262) and the National Basic Research Program of China (nos. 2007CB936203, 2011CB933003, and 2007CB310500).

\section{REFERENCES}

(1) Holt, J. K.; Park, H. G.; Wang, Y. M.; Stadermann, M.; Artyukhin, A. B.; Grigoropoulos, C. P.; Noy, A.; Bakajin, O. Science 2006, 312, 1034-1037.

(2) Majumder, M.; Chopra, N.; Andrews, R.; Hinds, B. J. Nature 2005, 438, 44-44.

(3) Thomas, J. A.; McGaughey, A. J. H. Nano Lett. 2008, $8,2788-2793$.

(4) Whitby, M.; Quirke, N. Nat. Nanotechnol. 2007, 2, 87-94.

(5) Thomas, J. A.; McGaughey, A. J. H. Phys. Rev. Lett. 2009, $102,184502$.

(6) Collins, P. C.; Arnold, M. S.; Avouris, P. Science 2001, 292, 706-709.

(7) Collins, P. G.; Hersam, M.; Arnold, M.; Martel, R.; Avouris, P. Phys. Rev. Lett. 2001, 86, 3128-3131.

(8) Na, P. S.; Kim, H. J.; So, H. M.; Kong, K. J.; Chang, H. J.; Ryu, B. H.; Choi, Y. M.; Lee, J. O.; Kim, B. K.; Kim, J. J.; Kim, J. H. Appl. Phys. Lett. 2005, 87, 093101.

(9) Maniwa, Y.; Matsuda, K.; Kyakuno, H.; Ogasawara, S.; Hibi, T.; Kadowaki, H.; Suzuki, S.; Achiba, Y.; Kataura, H. Nat. Mater. 2007, 6, 135-141.

(10) Jin, Z.; Chu, H. B.; Wang, J. Y.; Hong, J. X.; Tan, W. C.; Li, Y. Nano Lett. 2007, 7, 2073-2079.

(11) Yao, Y. G.; Li, Q. W.; Zhang, J.; Liu, R.; Jiao, L. Y.; Zhu, Y. T.; Liu, Z. F. Nat. Mater. 2007, 6, 283-286.

(12) Zheng, L. X.; O'Connell, M. J.; Doorn, S. K.; Liao, X. Z.; Zhao, Y. H.; Akhadov, E. A.; Hoffbauer, M. A.; Roop, B. J.; Jia, Q. X.; Dye, R. C.; Peterson, D. E.; Huang, S. M.; Liu, J.; Zhu, Y. T. Nat. Mater. 2004, 3, 673-676.

(13) Dresselhaus, M. S.; Dresselhaus, G.; Saito, R.; Jorio, A. Phys. Rep. 2005, 409, 47-99.

(14) Villalpando-Paez, F.; Son, H.; Nezich, D.; Hsieh, Y. P.; Kong, J.; Kim, Y. A.; Shimamoto, D.; Muramatsu, H.; Hayashi, T.; Endo, M.; Terrones, M.; Dresselhaus, M. S. Nano Lett. 2008, 8, 3879-3886.

(15) Yuan, Q. Z.; Zhao, Y. P. J. Am. Chem. Soc. 2009, 131, 6374-6376.

(16) Hummer, G.; Rasaiah, J. C.; Noworyta, J. P. Nature 2001, $414,188-190$.

(17) Wang, J.; Zhu, Y.; Zhou, J.; Lu, X. H. Phys. Chem. Chem. Phys. 2004, 6, 829-835.

(18) Washburn, E. W. Phys. Rev. 1921, 17, 273-283.

(19) Kuznetsova, A.; Popova, I.; Yates, J. T.; Bronikowski, M. J.; Huffman, C. B.; Liu, J.; Smalley, R. E.; Hwu, H. H.; Chen, J. G. G. J. Am. Chem. Soc. 2001, 123, 10699-10704.

(20) Chen, J. Y.; Kutana, A.; Collier, C. P.; Giapis, K. P. Science 2005, 310, 1480-1483.

(21) Mugele, F.; Baret, J. C. J. Phys.: Condens. Matter 2005, 17, R705-R774. 